\title{
Hyperthyroidism-Associated Insulin Resistance Is Not Mediated by Adiponectin Levels
}

\author{
Chih-Hsun Chu, ${ }^{1,2}$ Hing-Chung Lam, ${ }^{1,3}$ Jenn-Kuen Lee, ${ }^{1,3}$ Chih-Chen Lu, ${ }^{1}$ Chun-Chin Sun, ${ }^{1}$ \\ Mei-Chun Wang, ${ }^{1}$ and Ming-Ju Chuang ${ }^{1}$ \\ ${ }^{1}$ Division of Endocrinology and Metabolism, Department of Internal Medicine, Kaohsiung Veterans General Hospital, \\ 386, Ta-Chung 1st Road, Kaohsiung 813, Taiwan \\ ${ }^{2}$ Department of Rehabilitation Technology, TzuHui Institute of Technology, 367, San Min Road, Nanchou, Pingtung 926, Taiwan \\ ${ }^{3}$ School of Medicine, National Yang-Ming University, 155, Sec. 2, Li-Nong Street, Pei-Tou, Taipei 112, Taiwan
}

Correspondence should be addressed to Chih-Hsun Chu, chchu@isca.vghks.gov.tw

Received 8 September 2010; Revised 30 November 2010; Accepted 19 December 2010

Academic Editor: Gabriela Brenta

Copyright ( $\odot 2011$ Chih-Hsun Chu et al. This is an open access article distributed under the Creative Commons Attribution License, which permits unrestricted use, distribution, and reproduction in any medium, provided the original work is properly cited.

To evaluate the relationship between circulating adiponectin and insulin sensitivity in patients with hyperthyroid Graves' disease, we studied 19 adult patients with this disease and 19 age- and sex-matched euthyroid controls. All hyperthyroid patients were treated with antithyroid drugs and were re-evaluated after thyroid function normalized. Before antithyroid treatment, the adiponectin plasma concentrations were not different comparing with those in control group. The adiponectin levels remained unchanged after treatment. The homeostasis model assessment of insulin resistance (HOMA-IR) in hyperthyroid group was higher before treatment than after treatment. There was no significant difference in serum glucose and insulin levels between hyperthyroid and control groups and in the hyperthyroid group before and after treatment. BMI-adjusted adiponectin levels were not different among three groups. On the other hand, BMI-adjusted insulin levels and HOMA-IR values were significantly decreased after management of hyperthyroidism. Pearson's correlation revealed that insulin and HOMA-IR values positively correlated with triiodothyronine (T3) and free thyroxine (FT4) levels. However, adiponectin did not correlate with T3, FT4, insulin, HOMAIR and thyrotropin receptor autoantibody (TRAb) levels. In conclusion, insulin resistance associated with hyperthyroidism is not mediated by the levels of plasma adiponectin.

\section{Introduction}

Hyperthyroidism has been linked to reduced lean and fat body mass, resulting in lower-than-normal body weight. Patients with hyperthyroidism often have disrupted intermediary metabolism and thyrotoxicosis which has been associated with insulin resistance [1-3]. The mechanism of insulin resistance induced by thyrotoxicosis has not been completely elucidated. Adipocytokines which are regulated by thyroid hormones may play a role in the development of insulin resistance in hyperthyroidism.

Adipocytokines play crucial roles in the regulation of energy homeostasis, insulin sensitivity, lipid/carbohydrate metabolism, and even inflammatory/atherogenic reactions [4-11]. Among the adipocytokines, adiponectin is the only one to have anti-inflammatory and antiatherogenic properties [6-9]. It is paradoxically decreased in an insulin-resistance state [4]. We, therefore, measured plasma adiponectin concentrations in subjects with hyperthyroidism due to Graves' disease before and after antithyroid treatment and in euthyroid control subjects. The relationships among adiponectin, insulin concentrations, and homeostatic model assessment-insulin resistance index (HOMA-IR) were evaluated. We aimed to evaluate if adiponectin would be associated with the indicator of insulin resistance in patients with hyperthyroid Graves' disease.

\section{Methods}

2.1. Subjects. The study enrolled 19 patients with hyperthyroidism due to Graves' disease (14 women and 5 men) and 
TABLE 1: Characteristics of control subjects and hyperthyroid subjects before and after treatment.

\begin{tabular}{|c|c|c|c|}
\hline & \multirow[t]{2}{*}{ Control $(n=19)$} & \multicolumn{2}{|c|}{ Hyperthyroid $(n=19)$} \\
\hline & & Pretreatment & Posttreatment \\
\hline Age (years) & $36.7 \pm 2.7$ & $32.6 \pm 1.8$ & - \\
\hline $\operatorname{Sex}(F / M)$ & $17 / 2$ & $14 / 5$ & - \\
\hline Postmenopause & $3 / 17$ & $2 / 14$ & - \\
\hline Body Weight (kg) & $55.4 \pm 1.9$ & $53.9 \pm 1.8$ & $55.6 \pm 2.0$ \\
\hline $\operatorname{BMI}\left(\mathrm{kg} / \mathrm{m}^{2}\right)^{*}$ & $22.9 \pm 0.8$ & $20.6 \pm 0.5$ & $21.1 \pm 0.6$ \\
\hline Free T4 $(\mathrm{ng} / \mathrm{dL})^{*}$ & $1.35 \pm 0.05$ & $5.22 \pm 0.53$ & $1.14 \pm 0.09$ \\
\hline $\mathrm{T} 3(\mathrm{ng} / \mathrm{dL})^{*}$ & $113.1 \pm 5.3$ & $476.0 \pm 46.9$ & $115.6 \pm 8.0$ \\
\hline $\mathrm{TSH}(\mu \mathrm{IU} / \mathrm{mL})^{*}$ & $1.32 \pm 0.05$ & $0.02 \pm 0.00$ & $3.47 \pm 1.65$ \\
\hline Glucose $(\mathrm{mmol} / \mathrm{L})$ & $4.93 \pm 0.10$ & $5.14 \pm 0.08$ & $4.79 \pm 0.12$ \\
\hline Adiponectin (ng/L) & $6.55 \pm 0.71$ & $5.57 \pm 0.97$ & $6.62 \pm 0.80$ \\
\hline Insulin $(\mu \mathrm{IU} / \mathrm{mL})$ & $7.80 \pm 1.06$ & $8.86 \pm 1.02$ & $5.66 \pm 0.69$ \\
\hline HOMA-IR (mM mU/L)* & $1.71 \pm 0.23$ & $2.06 \pm 0.26$ & $1.21 \pm 0.16$ \\
\hline $\operatorname{TRAb}(\%)^{*}$ & - & $44.9 \pm 5.7$ & $29.1 \pm 5.0$ \\
\hline
\end{tabular}

pretreatment versus posttreatment, $P=.027$; control versus pre- or posttreatment, $P=$ ns.

19 age- and sex-matched euthyroid controls (17 women and 2 men) as previous study mentioned [12]. All hyperthyroid patients were treated with antithyroid drugs, either propylthiouracil (procil, Nysco Co., Ltd., Taiwan) or carbimazole (neo-thyreostat, Dr. Herbrand Co., Ltd., Germany). Thyroid function was normalized after three to seven months of treatment by antithyroid drugs (mean $=5.4 \pm 0.4$ months). The patients were evaluated at the time of diagnosis and after thyroid function normalized.

The plasma and serum samples were tested to measure serum concentrations of free thyroxine (FT4), total triiodothyronine (T3), thyrotropin (TSH), thyrotropin receptor autoantibody (TRAb), glucose, insulin, and plasma concentrations of adiponectin (the plasma assays of adiponectin had been performed simultaneously with the former protocol and not reported), all after overnight fasting. Body mass index (BMI) was calculated as weight in kilograms divided by the square of the height in meters. Insulin resistance was estimated by using the HOMA-IR index, calculated as serum glucose concentration $(\mathrm{mmol} / \mathrm{L}) \times$ serum insulin concentration $(\mathrm{mU} / \mathrm{L}) / 22.5$.

2.2. Biochemistry and Hormone Analyses. Serum glucose, FT4, T3, TSH, and insulin concentrations were measured as former protocol reported [12]. Plasma adiponectin concentrations were measured by the quantitative sandwich enzyme immunoassay technique ( R \& D systems, Inc., Minneapolis, $\mathrm{MN}, \mathrm{USA})$. The intraassay and inter-assay CVs were $3.4 \%$ and $5.8 \%$, respectively. The sensitivity of the assay was $0.246 \mathrm{ng} / \mathrm{mL}$. Serum TRAb was analyzed by radioreceptor assay method (RSR Ltd., Cardiff CF23 8HE, UK). The levels greater than $10 \%$ was considered positive.
2.3. Statistical Analyses. Data were reported as mean \pm standard error of the mean (SEM). Comparisons of hyperthyroid subjects before treatment, hyperthyroid subjects after treatment, and euthyroid control subjects were made by using one-way analysis of variance (ANOVA) and Bonferroni test for post-hoc multiple comparisons. Mann-Whitney test was used for nonparametric data. Correlations between parameters were assessed by using Pearson's correlation analysis. A value of $P<.05$ was considered as statistically significant.

\section{Results}

The demographic and clinical characteristics of the study population are shown in Table 1 . The mean \pm SEM age was $32.6 \pm 1.8$ years for hyperthyroid subjects and $36.7 \pm 2.7$ years for matching control subjects. As expected, subjects in the hyperthyroid group before treatment had lower TSH and higher T3 and FT4 serum concentrations than they did after treatment or the control group. In addition, TRAb also decreased after antithyroid drugs treatment $(44.9 \pm 5.7$ versus $29.1 \pm 5.0 \%$ ).

HOMA-IR values were higher in hyperthyroid group before treatment $(2.06 \pm 0.26 \mathrm{mM} \mathrm{mU} / \mathrm{L})$ than after treatment $(1.21 \pm 0.16 \mathrm{mM} \mathrm{mU} / \mathrm{L}, P=.027)$. Before antithyroid treatment, the adiponectin plasma concentrations were not different comparing with those in control group (5.57 \pm 0.97 versus $6.55 \pm 0.71 \mathrm{ng} / \mathrm{L}$, ns). The adiponectin levels remained unchanged after treatment $(6.62 \pm 0.80$ versus $5.57 \pm 0.97 \mathrm{ng} / \mathrm{L}, \mathrm{ns})$. There was no significant difference in serum glucose, and insulin levels between hyperthyroid and control groups and in the hyperthyroid group before and after treatment. 
TABLE 2: Comparisons of BMI-adjusted plasma adiponectin concentration, insulin concentration, and HOMA-IR value among the control and hyperthyroid groups before and after treatment.

\begin{tabular}{lccr}
\hline & Control $(n=19)$ & & Hyperthyroid \\
& & Pretreatment $(n=19)$ & Posttreatment $(n=19)$ \\
\hline Adiponectin/BMI & $0.30 \pm 0.02$ & $0.28 \pm 0.05$ & $0.32 \pm 0.04$ \\
Insulin/BMI* & $0.33 \pm 0.04$ & $0.43 \pm 0.05$ & $0.26 \pm 0.03$ \\
HOMA-IR/BMI* & $0.07 \pm 0.01$ & $0.10 \pm 0.01$ & $0.06 \pm 0.01$ \\
\hline
\end{tabular}

Data are mean \pm SEM. Comparisons among three groups were made by one-way analysis of variance. $* P<.05$. Post-hoc test by Bonferroni; Insulin/BMI: pretreatment versus posttreatment, $P=.010$. HOMA-IR/BMI: pretreatment versus posttreatment, $P=.005$.

TABle 3: Pearson's correlation coefficients in patients with hyperthyroidism due to Graves' disease.

\begin{tabular}{lccc}
\hline Factors & Adiponectin & Insulin & HOMA-IR \\
\hline BMI & -0.197 & 0.219 & 0.207 \\
T3 & -0.024 & $0.328^{*}$ & $0.364^{*}$ \\
FT4 & 0.141 & $0.405^{*}$ & $0.444^{* *}$ \\
Adiponectin & 1 & -0.065 & -0.095 \\
Insulin & -0.065 & 1 & $0.991^{* *}$ \\
HOMA-IR & -0.095 & $0.991^{* *}$ & 1 \\
TRAb & 0.170 & 0.099 & 0.098 \\
\hline$P<.05, * * P<.01$. & & &
\end{tabular}

BMI-adjusted adiponectin levels were not different among three groups (Table 2). On the other hand, BMIadjusted insulin levels and HOMA-IR values were significantly decreased after management of hyperthyroidism.

Pearson's correlation (Table 3 ) revealed that insulin and HOMA-IR values were positively correlated with T3 and FT4 levels. However, adiponectin did not correlate with T3, FT4, insulin, HOMA-IR, and TRAb levels.

\section{Discussion}

Patients with hyperthyroidism are known to have elevated fasting serum glucose levels $[13,14]$, which may be explained by increased endogenous glucose production through more rapid glycogenolysis and gluconeogenesis $[13,15,16]$. The speed of insulin-stimulated glucose disposal in peripheral tissues is variable in hyperthyroidism and may be normal, increased, or decreased [3].

Adipose tissue, an endocrine active tissue, releases adipocytokines to modulate several peripheral tissue functions. Among these adipocytokines, adiponectin is a 244 amino acid protein which is also known as the adipose most abundant gene transcript 1 . Reduction in adiponectin secretion has been found to be associated with the development of insulin resistance in animal models of obesity and lipoatrophy [4]. Injection of adiponectin into nonobese diabetic mice may lead to an insulin-independent decrease in glucose levels [17]. Plasma adiponectin concentrations in patients with both obesity and type 2 diabetes mellitus were found to be reduced $[18,19]$. Adiponectin and thyroid hormones share some physiological actions as reduction of body fat by increasing thermogenesis and lipid oxidation.

One animal study had showed a potential inhibitory effect of T3 on adiponectin mRNA expression on adipose tissues [20]. However, adiponectin concentrations had also been found to be associated with FT4 levels in patients with hypothyroidism [21]. The effect of thyroid hormones on the adiponectin levels revealed contrary results, which needs more study to clarify.

From the limited studies on the relationship between circulating adiponectin levels and thyroid function status, results have been inconsistent. In human study, hyperthyroidism has been associated with similar $[22,23]$ or elevated $[24,25]$ circulating adiponectin levels compared with euthyroid subjects. Normalization of hyperthyroidism with appropriate therapy was not accompanied by significant changes in circulating adiponectin levels in one study [22]. Conversely, in another study a significant reduction was found [26]. In addition, this study also demonstrated that hyperadiponectinemia was associated with levels of TRAb, pointing to an immunological influence on adiponectin metabolism. However, we did not find correlation between $\mathrm{TRAb}$ and adiponectin levels in hyperthyroid patients.

In the present study, HOMA-IR values and BMI-adjusted insulin concentrations were decreased after hyperthyroidism management. In addition, HOMA-IR and insulin levels were positively correlated with T3 and FT4 levels in patients with Graves' disease. These findings were consistent with the assumption that hyperthyroidism is associated with higher insulin resistance. However, we did not observe difference in adiponectin concentrations among hyperthyroid and control patients and also the posttreatment patients. There was no correlation between serum insulin or HOMA-IR levels and plasma adiponectin concentrations. These observations suggest that insulin resistance associated with hyperthyroidism is not mediated by a reduction in plasma adiponectin levels. However, because of the small sample, these results must be interpreted with caution.

Hyperthyroidism could alter the neurophysiology of food intake regulation which may drive the marked hyperphagia and craving for carbohydrates [27]. The carbohydrate-rich diet with a high glycemic load appears to be associated with lower adiponectin concentrations [28]. Therefore, the carbohydrates intake in hyperthyroid patients may influence adiponectin levels. 
Hyperthyroidism is associated with insulin resistance. However, hyperthyroidism also reduces BMI, which may increase insulin sensitivity. Plasma adiponectin concentrations were decreased in patients with obesity. Besides, the levels also paradoxically decreased in an insulin-resistance state. The concentrations of adiponectin among hyperthyroid subjects may be influenced by many conflicting factors. However, no study has reported a decreased adiponectin concentration in hyperthyroidism. Therefore, the insulin resistance associated with thyrotoxicosis is likely not to be mediated by altered adiponectin secretion.

\section{Conclusions}

In conclusion, insulin resistance associated with hyperthyroidism is not mediated by the levels of plasma adiponectin. Additional studies are needed to clarify the contribution of these adipocytokines in the development of insulin resistance in patients with hyperthyroidism.

\section{Conflict of Interests}

All authors have no conflict of interests to declare.

\section{References}

[1] A. P. Weetman, “Graves' disease," New England Journal of Medicine, vol. 343, no. 17, pp. 1236-1248, 2000.

[2] L. H. Duntas, "Thyroid disease and lipids," Thyroid, vol. 12, no. 4, pp. 287-293, 2002.

[3] G. D. Dimitriadis and S. A. Raptis, "Thyroid hormone excess and glucose intolerance," Experimental and Clinical Endocrinology and Diabetes, vol. 109, no. 2, pp. S225-S239, 2001.

[4] T. Yamauchi, J. Kamon, H. Waki et al., "The fat-derived hormone adiponectin reverses insulin resistance associated with both lipoatrophy and obesity," Nature Medicine, vol. 7, no. 8, pp. 941-946, 2001.

[5] C. S. Mantzoros, "The role of leptin in human obesity and disease: a review of current evidence," Annals of Internal Medicine, vol. 130, no. 8, pp. 671-680, 1999.

[6] H. Chen, M. Montagnani, T. Funahashi, I. Shimomura, and M. J. Quon, "Adiponectin stimulates production of nitric oxide in vascular endothelial cells," Journal of Biological Chemistry, vol. 278, no. 45, pp. 45021-45026, 2003.

[7] N. Ouchi, S. Kihara, Y. Arita et al., "Adipocyte-derived plasma protein, adiponectin, suppresses lipid accumulation and class A scavenger receptor expression in human monocyte-derived macrophages," Circulation, vol. 103, no. 8, pp. 1057-1063, 2001.

[8] N. Ouchi, S. Kihara, T. Funahashi et al., "Reciprocal association of C-reactive protein with adiponectin in blood stream and adipose tissue," Circulation, vol. 107, no. 5, pp. 671-674, 2003.

[9] M. Kumada, S. Kihara, N. Ouchi et al., "Adiponectin specifically increased tissue inhibitor of metalloproteinase-1 through interleukin-10 expression in human macrophages," Circulation, vol. 109, no. 17, pp. 2046-2049, 2004.

[10] W. Aldhahi and O. Hamdy, "Adipokines, inflammation, and the endothelium in diabetes," Current Diabetes Reports, vol. 3, no. 4, pp. 293-298, 2003.
[11] A. Fukuhara, M. Matsuda, M. Nishizawa et al., "Visfatin: a protein secreted by visceral fat that Mimics the effects of insulin," Science, vol. 307, no. 5708, pp. 426-430, 2005.

[12] C. H. Chu, J. K. Lee, M. C. Wang et al., "Change of visfatin, C-reactive protein concentrations, and insulin sensitivity in patients with hyperthyroidism," Metabolism, vol. 57, no. 10, pp. 1380-1383, 2008.

[13] A. Wennlund, P. Felig, L. Hagenfeldt, and J. Wahren, "Hepatic glucose production and splanchnic glucose exchange in hyperthyroidism," Journal of Clinical Endocrinology and Metabolism, vol. 62, no. 1, pp. 174-180, 1986.

[14] M. J. Holness and M. C. Sugden, "Continued glucose output after re-feeding contributes to glucose intolerance in hyperthyroidism," Biochemical Journal, vol. 247, no. 3, pp. 801-804, 1987.

[15] M. J. Muller, U. Paschen, and H. J. Seitz, "Thyroid hormone regulation of glucose homeostasis in the miniature pig," Endocrinology, vol. 112, no. 6, pp. 2025-2031, 1983.

[16] M. P. Sandler, R. P. Robinson, D. Rabin et al., "The effect of thyroid hormones on gluconeogenesis and forearm metabolism in man," Journal of Clinical Endocrinology and Metabolism, vol. 56, no. 3, pp. 479-485, 1983.

[17] A. H. Berg, T. P. Combs, X. Du, M. Brownlee, and P. E. Scherer, "The adipocyte-secreted protein Acrp30 enhances hepatic insulin action," Nature Medicine, vol. 7, no. 8, pp. 947953, 2001.

[18] Y. Arita, S. Kihara, N. Ouchi et al., "Paradoxical decrease of an adipose-specific protein, adiponectin, in obesity," Biochemical and Biophysical Research Communications, vol. 257, no. 1, pp. 79-83, 1999.

[19] K. Hotta, T. Funahashi, Y. Arita et al., "Plasma concentrations of a novel, adipose-specific protein, adiponectin, in type 2 diabetic patients," Arteriosclerosis, Thrombosis, and Vascular Biology, vol. 20, no. 6, pp. 1595-1599, 2000.

[20] A. Cabanelas, A. Cordeiro, N. A. D. Santos Almeida et al., "Effect of triiodothyronine on adiponectin expression and leptin release by white adipose tissue of normal rats," Hormone and Metabolic Research, vol. 42, no. 4, pp. 254-260, 2010.

[21] S. Y. Lin, S. C. Huang, and W. H. H. Sheu, "Circulating adiponectin concentrations were related to free thyroxine levels in thyroid cancer patients after thyroid hormone withdrawal," Metabolism, vol. 59, no. 2, pp. 195-199, 2010.

[22] P. Iglesias, P. Alvarez Fidalgo, R. Codoceo, and J. J. Díez, "Serum concentrations of adipocytokines in patients with hyperthyroidism and hypothyroidism before and after control of thyroid function," Clinical Endocrinology, vol. 59, no. 5, pp. 621-629, 2003.

[23] F. Santini, A. Marsili, C. Mammoli et al., "Serum concentrations of adiponectin and leptin in patients with thyroid dysfunctions," Journal of Endocrinological Investigation, vol. 27, no. 2, pp. RC5-RC7, 2004.

[24] S. Yaturu, S. Prado, and S. R. Grimes, "Changes in adipocyte hormones leptin, resistin, and adiponectin in thyroid dysfunction," Journal of Cellular Biochemistry, vol. 93, no. 3, pp. 491496, 2004.

[25] L. Sieminska, D. Niedziolka, A. Pillich et al., "Serum concentrations of adiponectin and resistin in hyperthyroid Graves' disease patients," Journal of Endocrinological Investigation, vol. 31, no. 9, pp. 745-749, 2008.

[26] T. Saito, T. Kawano, T. Saito et al., "Elevation of serum adiponectin levels in Basedow disease," Metabolism, vol. 54, no. 11, pp. 1461-1466, 2005. 
[27] H. Pijl, P. H. E. M. de Meijer, J. Langius et al., "Food choice in hyperthyroidism: potential influence of the autonomic nervous system and brain serotonin precursor availability," Journal of Clinical Endocrinology and Metabolism, vol. 86, no. 12, pp. 5848-5853, 2001.

[28] T. Pischon, C. J. Girman, N. Rifai, G. S. Hotamisligil, and E. B. Rimm, "Association between dietary factors and plasma adiponectin concentrations in men," American Journal of Clinical Nutrition, vol. 81, no. 4, pp. 780-786, 2005. 


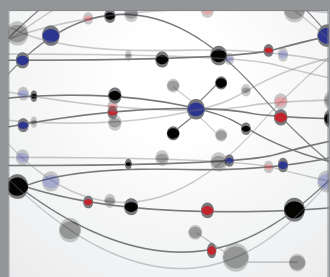

The Scientific World Journal
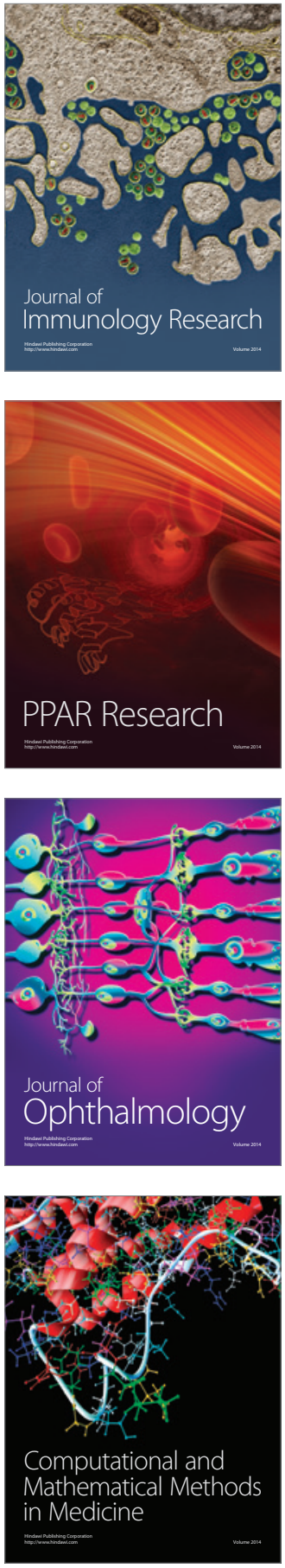

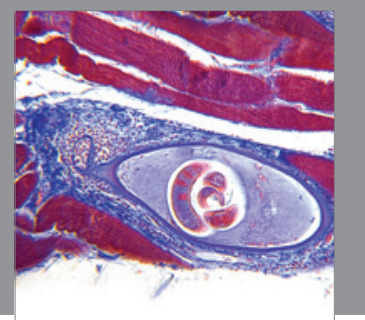

Gastroenterology

Research and Practice
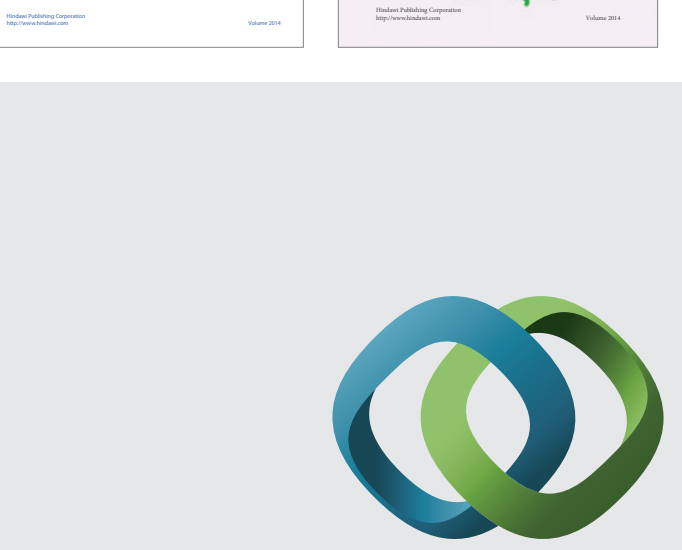

\section{Hindawi}

Submit your manuscripts at

http://www.hindawi.com
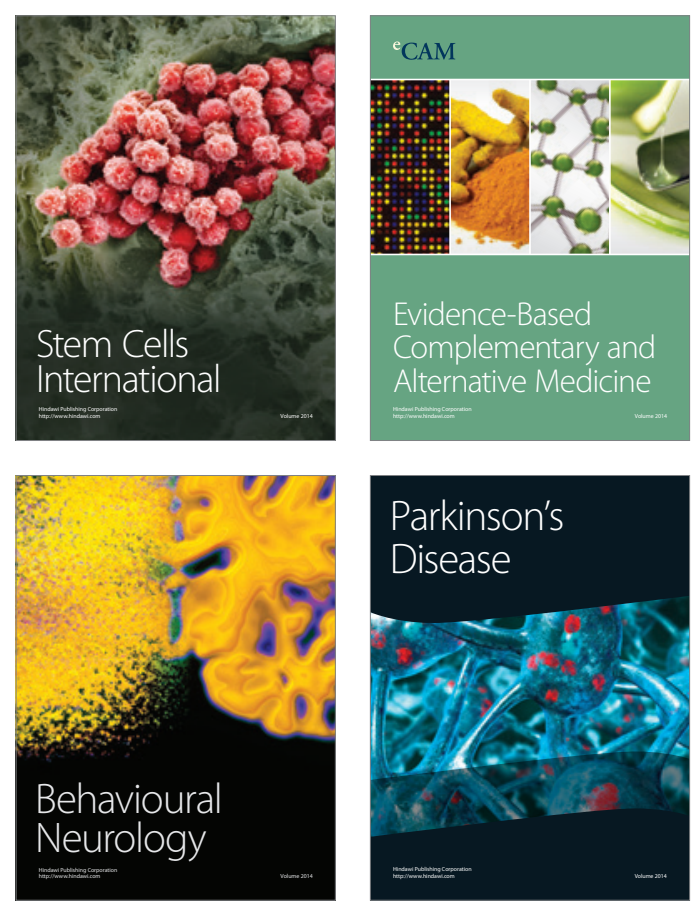

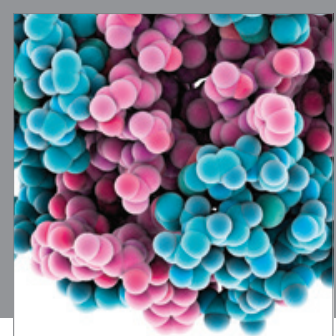

Journal of
Diabetes Research

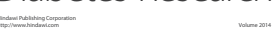

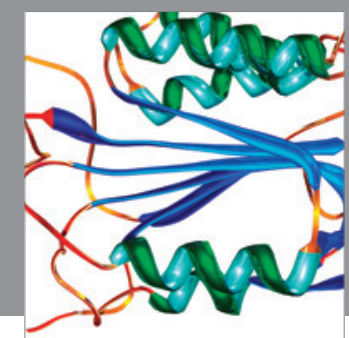

Disease Markers
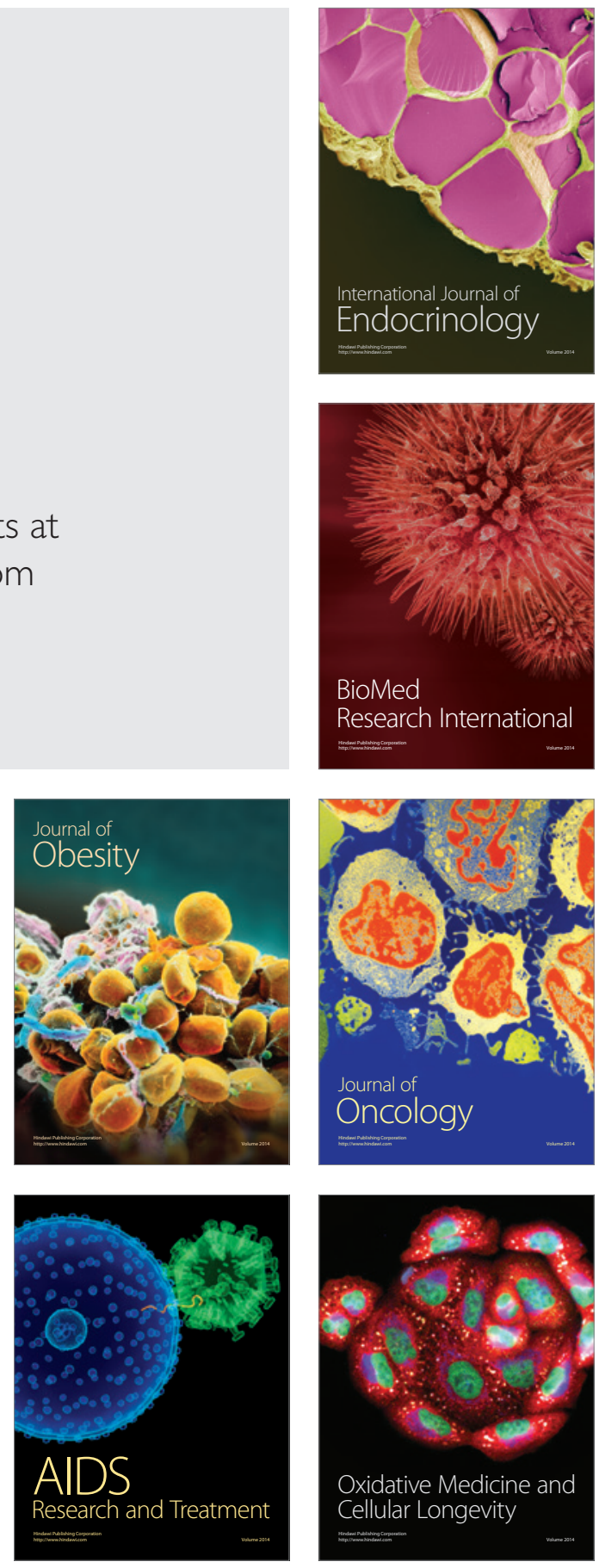